\title{
Surface and bulk core level study of PdTe using HAXPES
}

Cite as: AIP Conference Proceedings 2115, 030339 (2019); https://doi.org/10.1063/1.5113178

Published Online: 12 July 2019

Ram Prakash Pandeya, Arindam Pramanik, Pramita Mishra, A. Thamizhavel, and Kalobaran Maiti

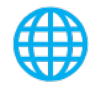

\section{ARTICLES YOU MAY BE INTERESTED IN}

Preparation and electronic structure study of a topological crystalline insulator, SnTe AIP Conference Proceedings 2115, 030343 (2019); https://doi.org/10.1063/1.5113182

Preparation of high quality $\mathrm{Cr}$ films on W(100) surface

AIP Conference Proceedings 2115, 030341 (2019); https://doi.org/10.1063/1.5113180

The quantum spin Hall effect and topological insulators

Physics Today 63, 33 (2010); https://doi.org/10.1063/1.3293411

\section{Challenge us.}

What are your needs for periodic signal detection?

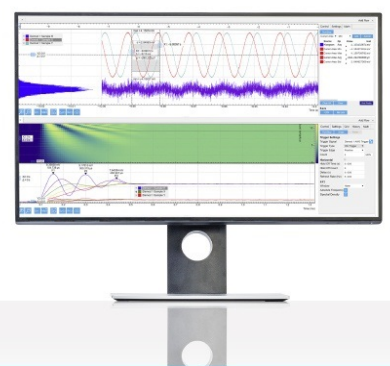

- Zurich - Instruments 


\title{
Surface and bulk core level study of PdTe using HAXPES
}

\author{
Ram Prakash Pandeya, Arindam Pramanik, Pramita Mishra, A. Thamizhavel and \\ Kalobaran Maiti ${ }^{a}$
}

\author{
Department of Condensed Matter Physics and Material Science, Tata Institute of Fundamental Research, Homi \\ Bhabha Road, Colaba, Mumbai - 400005, India. \\ ${ }^{\text {a} C}$ Corresponding author: kbmaiti@tifr.res.in
}

\begin{abstract}
We report here the preparation of high quality single crystal of PdTe(0001). Various characterizations show good quality of the sample formed in single phase with large sample size suitable for various spectroscopic measurements. We have carried out hard $x$-ray photoemission spectroscopic (HAXPES) measurements and found interesting results. While Te core level spectrum shows asymmetry as expected in a metallic system, PdTe, the Pd core spectrum does not show such features indicating element selective behavior in the photoemission spectra. We also observe satellites in the core level spectra providing evidence for finite electron correlation induced effect in the electronic structure. Surface and bulk electronic structure is found to be similar in this system.
\end{abstract}

\section{INTRODUCTION}

Superconductors are generally classified by two broad categories - (i) conventional superconductors; properties of these materials can be captured by Bardeen-Cooper-Schrieffer (BCS) theory and (ii) unconventional superconductors, where BCS theory is not adequate to capture their electronic properties. Most of the high temperature superconductors such as cuprates and Fe-based systems belong to the unconventional type. Despite extensive research, the origin of superconductivity in unconventional superconductors is still a puzzle. The major concern is the finding of the glue that sticks two electrons together forming an electron pair necessary for the superconducting ground state. Fe-based systems exhibit additional complexity due to the discovery of coexisting magnetic order and superconductivity as well as complex structural phase diagram. It is understood that Fe-based systems can be classified as unconventional superconductors. Among the binary compounds, FeSe exhibits superconductivity at $6 \mathrm{~K}$. FeTe is an antiferromagnetic metal. Interestingly, superconducting transition temperature becomes much larger than that of $\mathrm{FeSe}$ in $\mathrm{FeTe}_{1-x} \mathrm{Se}_{x}$ where disorder is expected to play significant role. While the superconductivity in all these materials is of unconventional type, PdTe is an example in the same class, where the superconductivity is of conventional type.

PdTe is a type-II superconductor with transition temperature close to $4.2 \mathrm{~K}$. Theoretical calculation shows strong hybridization between $\mathrm{Pd} 4 d$ and Te $5 p$ orbitals, which is similar to many other transition metal chalcogenide and pnictide unconventional superconductors. It forms in a hexagonal structure with space group, $\boldsymbol{P} 6_{3} / \mathrm{mmc}$. There are some reports on the synthesis of PdTe in its stoichiometric ratio and physical property measurements on it. To our knowledge, most of the measurements reported so far are done on polycrystalline samples of PdTe [1-3]. To study the anisotropy in bulk properties, electronic structure, topology of the gaps, etc. one requires very good quality single crystal. Here, we report our preparation of single crystalline PdTe and its characterization by various techniques. We have also performed hard $x$-ray photoelectron emission spectroscopy to study the electronic structure of the material. Surface sensitivity of the technique has been varied to capture the differences/similarities between the bulk and surface electronic structure of PdTe. 


\section{EXPERIMENTAL METHOD}

Single crystal of PdTe was grown by melting the mixture of small pieces of high purity Pd (99.95\%) wire and the chunks of Te (99.999\%). To minimize the external impurity inside the sample, we sealed the $\mathrm{Pd}$ and Te mixture in the ratio of $1: 1$ in a quartz ampule at a vacuum of $10^{-6}$ Torr. The sealed ampule was heated in a box furnace to $1000{ }^{\circ} \mathrm{C}$; the temperature was brought up at a rate of $60^{\circ} \mathrm{C} / \mathrm{hr}$ and then it was kept at $1000^{\circ} \mathrm{C}$ for 3 days. After that the ampule temperature was reduced very slowly $\left(2{ }^{\circ} \mathrm{C} / \mathrm{hr}\right)$ to $650{ }^{\circ} \mathrm{C}$ and then it was cooled to room temperature at a rate of 60 ${ }^{\circ} \mathrm{C} / \mathrm{hr}$. After 12 days of this process, we were able to get very big and shiny single crystals of PdTe. The characterization of this sample was done by employing powder $x$-ray diffraction (XRD), Laue XRD and energy dispersive analysis of $x$-rays (EDX). The powder XRD measurement was carried out using Panalytical XPERT-PRO vs. 2.1E $x$-ray diffractometer.

To study the surface and bulk electronic structure, we have performed high resolution hard $x$-ray photoemission spectroscopy (HAXPES) at two different emission angels for same photon energy. This measurement was carried out at P09 beamline at Petra III synchrotron radiation center, DESY Hamburg, Germany. The photon energy used to excite the electrons was $5946 \mathrm{eV}$. The HAXPES measurements were carried out at $32 \mathrm{~K}$ temperature. The sample was cleaved in situ to expose the clean sample surface for measurements. Core level spectral fitting was done using CasaXPS software.

\section{RESULTS AND DISCUSSIONS}

In Fig. 1(a), we show the experimentally measured XRD pattern (open black circle) exhibiting sharp, intense and distinct peaks for different reflections. The pattern is consistent with earlier results [1-3] and establish single phase of the material prepared. The inset of Fig. 1(a) shows the unit cell of PdTe - red and black solid spheres are Pd and Te

(a)

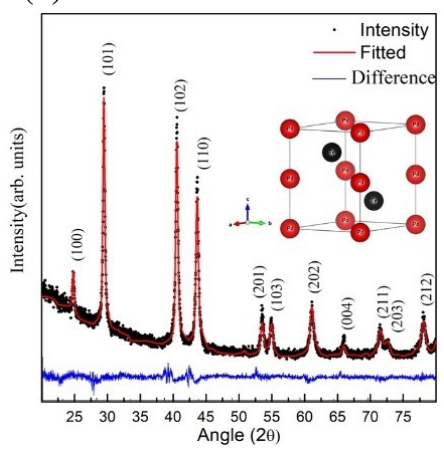

(b)

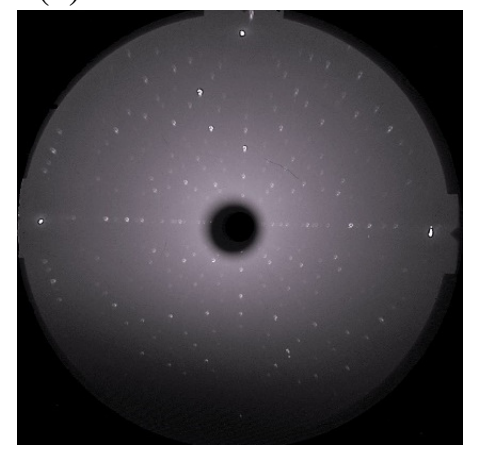

(c)

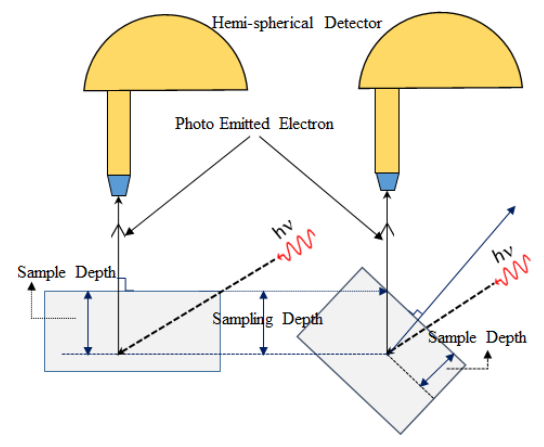

FIGURE 1. (a) Powder $x$-ray diffraction pattern. The inset figure is the unit cell of PdTe. (b) Laue X-ray back diffraction pattern of PdTe(0001) single crystal. (c) Experimental setup for HAXPES study.

atoms, respectively. Using the Wyckoff positions of each atom, we generated the Full Prof XRD data fitting the experimental results. The fit data superimposed over the experimental results exhibit an excellent representation; the difference between the experimental and simulated data is negligibly small. The lattice parameters extracted from fitting are $a=b=4.1471( \pm 0.0006) \AA$ and $c=5.665( \pm 0.001) \AA$ with $\mathrm{Chi}^{2}$ value of 1.85 . The elemental analysis of PdTe was carried out employing EDX analysis. The Pd:Te ratio obtained from EDX is found very close to 1:1. We have also performed back reflection Laue XRD on this sample. In Fig. 1(b), we show the Laue XRD pattern of PdTe for the (0001) plane of the crystal. Similar pattern was observed from the Laue XRD simulation using software OrientExpress V3.3 indicating good crystallinity of the sample. 
HAXPES measurements were carried out at two experimental geometries to vary the surface sensitivity [4,5] of the technique - (i) normal emission (emission angle kept at $10^{\circ}$ with the surface normal, which is close to normal emission) and (ii) angled emission (emission angle $=60^{\circ}$ ).

The experimental setup is shown in Fig. 1(c). It is clear that if $\lambda$ is the mean escape depth (in figure it is the sampling depth) of the photoelectrons for normal emission geometry, the probing depth becomes $\lambda / 2$ for $60^{\circ}$ angled emission and hence it becomes significantly more surface sensitive at angled emission geometry. In this way, one can probe both surface and bulk electronic structure without changing the scattering cross section which can be a big issue if one changes the photon energy to probe the surface and bulk electronic structure.

In Fig. 2, we show both the normal and $60^{\circ}$ angled emission spectra of Pd $3 p$ and Te $3 d$ core levels at $32 \mathrm{~K}$ temperature. The spin-orbit split $\mathrm{Pd} 3 p_{3 / 2}$ and $3 p_{1 / 2}$ peaks appear at $533.1 \mathrm{eV}$ and $560.2 \mathrm{eV}$, respectively with a spin orbit splitting of about $27.1 \mathrm{eV}$. Te $3 d_{5 / 2}$ and $3 d_{3 / 2}$ peaks appear at $573 \mathrm{eV}$ and $583.3 \mathrm{eV}$, respectively (spin orbit splitting is about $10.3 \mathrm{eV}$ ). The binding energy of Te $3 d_{5 / 2}$ and $\mathrm{Te} 3 d_{3 / 2}$ are close to the binding energy for the same core levels in pure Te, whereas for Pd $3 p$ state binding energy is larger than the same state in pure Pd by about $0.8 \mathrm{eV}$ [6]. One reason for such a large shift could be significant positive charge/Madelung potential at $\mathrm{Pd}$ sites although Te seems to be close to neutral. This is unusual. The other reason could be less efficient screening of $\mathrm{Pd}$ core holes in the photoemission final state while the Te core holes are screened well. In addition, we observe several weak features, which could be due to the final state effects of the photoexcitation process.

In order to find the constituent features in the spectra,

(a)

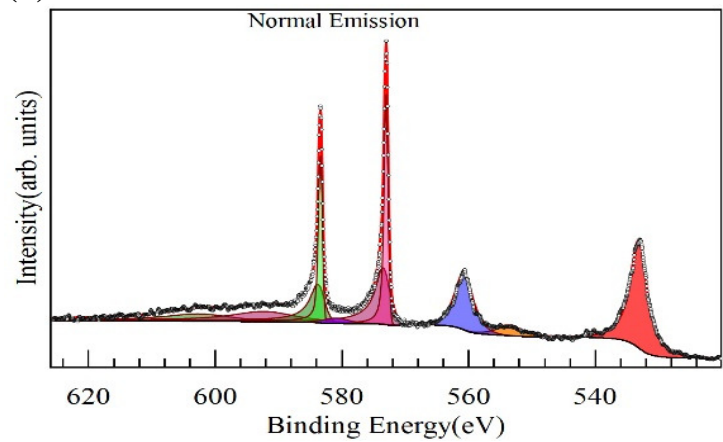

(b)

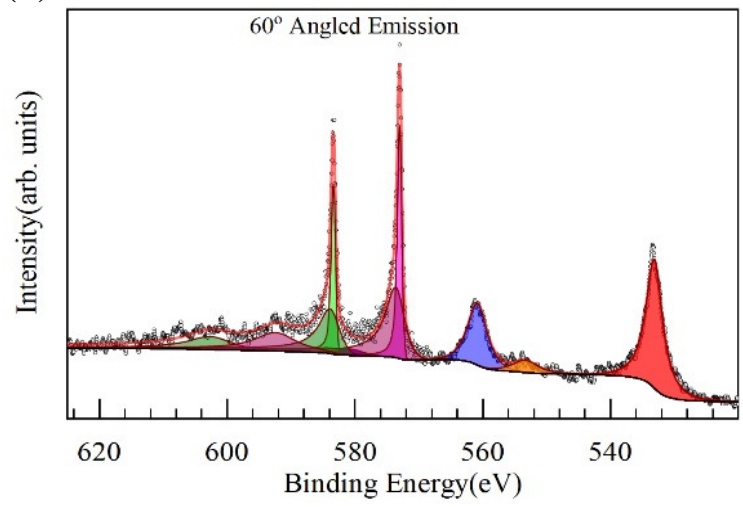

FIGURE 2. Pd $3 p$ and Te $3 d$ core level HAXPES spectra at $32 \mathrm{~K}$ temperature. (a) Normal emission. (b) $60^{\circ}$ angled emission. the whole energy region probed is fitted with 10 peaks; each of the peaks correspond to distinct feature in the spectra. These features are asymmetric Gaussian-Lorentzian (GL) product function. Interestingly, the Gaussian part is found to be arising primarily from the resolution of the experiment and the intense features can be captured well by varying only the Lorentzian width, which represent the lifetime broadening of the photo hole and photoelectrons. GL mixing ratio for the weak intensities, however, could be varied. For a metallic system, it is expected that the core level peaks will show asymmetry towards higher binding energies due to low energy excitations across the Fermi level. Since, PdTe is a metal, one expects similar behavior in all core level spectra. Interestingly, both main and satellite peaks of $\operatorname{Pd} 3 p$ could be captured well without considering asymmetry, while fitting of Te $3 d$ requires consideration of asymmetry.

The energy separation and intensity ratio of the weak features indicate that they are associated to the photoexcitations of $\mathrm{Pd} 3 p$ and Te $3 d$ core level electrons. The binding energy difference between the Pd $3 p$ main peak and satellite peak is $27.6 \mathrm{eV}$ and indicate significant electron correlation induced effect for the Pd electrons. Te $3 d$ core level features also shows satellite peaks. In this case, it is fitted with two satellite peaks; binding energy of Te $3 d_{5 / 2}$ satellites states are $573.7 \mathrm{eV}$ and $592.7 \mathrm{eV}$. There are quite a few papers on the theoretically calculated electronic structure of bulk PdTe but most of them have not taken the electron correlation term into account [2,3,7]. Presence of satellite peaks in bulk PdTe core level spectra indicates that the electron-electron interaction is significant and one needs to consider the correlation induced effect in the electronic structure of PdTe.

Similar effects can also be seen in $60^{\circ}$ angled emission spectrum shown in Fig. 2(b). There is no change in peak position and asymmetry of either main or satellite peaks with change of emission angle indicating that the surface and 
bulk electronic structure are very similar in this system. Interestingly, the full width at half maxima (FWHM) for some of the peaks changes with angle. In earlier studies, it was found that the core level spectra related to the surface of the sample either have no change or more broadening effect in comparison to the bulk core level peaks [8-13]. In our study, FWHM of the core level peaks in angled emission spectrum are close to the normal emission data, except the second satellite observed for Te $3 d$ core level signals; width of this peak decreased in the angled emission spectrum. Phonon broadening of bulk core level states for a monoatomic lattice system was first formulated by the Overhauser [13]. According to his theory, the bulk core level state broadening linearly depends on the fourth power of (T/ $\left.\theta_{D}\right)$ where $\theta_{D}$ is the Debye temperature of the solid. Later Wertheim et al. showed that $\theta_{D}$ for surface is less than the bulk $\theta_{D}$ value and used Overhauser's theory to calculate the change in FWHM for the surface core level states [13]. Clearly, our observation of decrease in FWHM in the more surface sensitive spectrum is not in line with this theory. Moreover, the energy scale of the change is in $\mathrm{eV}$, which suggest that other considerations are necessary to explain these observations.

\section{CONCLUSIONS}

In conclusion, we have grown high quality single crystals of PdTe, which could be utilized for spectroscopic investigations. We have also performed the hard $x$-ray photoemission spectroscopy on PdTe (0001). This measurement was carried out at two different emission angles to probe the differences between the bulk and surface electronic structure. While Te $3 d$ spectra exhibit asymmetry in the core level lineshape as expected in a metallic system, Pd $3 p$ core level spectra do not show asymmetry in both normal emission and angled emission measurements suggesting element specific electron dynamics. Presence of satellite peaks in both Pd and Te core level spectra indicates finite electron correlation induced effect for both $\mathrm{Pd}$ and Te electrons, which is mostly ignored in many theoretical electronic band structure calculations. Change in FWHM of Te $3 d$ satellite shows opposite trend to the phonon broadening theory with change of photoelectron emission angle.

\section{ACKNOWLEDGMENTS}

The authors acknowledge financial support from the DST-DESY project to perform the experiments at P09 beamline at PETRA III, Hamburg, Germany and Dr. Indranil Sarkar for his help during the measurements.

\section{REFERENCES}

1. Amar B. Kakri et al., J.Phys., Condens. Matter 24(5), 055701 (2012).

2. Amar B. Kakri et al., PNAS 110 23, 9287 (2013).

3. B. Tiwari et al., Supercond. Sci. Technol. 28, 055008 (2015).

4. K. Maiti et al., Phys. Rev. B 73, 052508 (2006).

5. S. Thakur, K. Maiti, Solid State Commun. 193, 45 (2014).

6. X-ray Data Booklet, Center of X-ray Optics and Advanced Light Source, Lawrence Berkeley National Laboratory(website:- http://xdb.lbl.gov/).

7. Chindedu E Ekuma et al., J. Phys.: Condes. Matter 25, 405601 (2013).

8. Jin-Jin Cao, Xiao-Fan Gou, Physica C: Superconductivity and its applications 520, 19-23 (2016).

9. D. M. Riffe and G. K. Wertheim, Phys.Rev. B 47, 6672 (1993).

10. G. K. Wertheim and D. N. E. Bunchanan, Phys. Rev. B 43, 13815 (1991).

11. G. K. Wertheim et al., Phys. Rev. B 30, 4343 (1984).

12. L. I. Johansson,P.-A. Glans and T. Balasubramanian, Phys. Rev. B 58, 3621 (1998).

13. D. M. Riffe and G. K. Wertheim, Phys. Rev. B 61, 3 (2000). 